\title{
Mining Multidimensional Contextual Outliers from Categorical Relational Data .
}

\author{
Guanting Tang \\ School of Computing Science \\ Simon Fraser University \\ gta9@cs.sfu.ca \\ James Bailey \\ Department of Computing and \\ Information Systems \\ The University of Melbourne \\ baileyj@unimelb.edu.au
}

\author{
Jian Pei \\ School of Computing Science \\ Simon Fraser University \\ jpei@cs.sfu.ca \\ Guozhu Dong \\ Department of Engineering \\ and Computer Science \\ Wright State University \\ guozhu.dong@wright.edu
}

\begin{abstract}
A wide range of methods have been proposed for detecting different types of outliers in full space and subspaces. However, the interpretability of outliers, that is, explaining in what ways and to what extent an object is an outlier, remains a critical open issue. In this paper, we develop a notion of contextual outliers on categorical data. Intuitively, a contextual outlier is a small group of objects that share strong similarity with a significantly larger reference group of objects on some attributes, but deviate dramatically on some other attributes. We develop a detection algorithm, and conduct experiments to evaluate our approach.
\end{abstract}

\section{Keywords}

Outlier detection, context, categorical data

\section{INTRODUCTION}

Outlier detection is an important data mining task. A wide range of methods have been proposed to detect different types of outliers on various kinds of data. Interpretability of outliers, however, remains a serious concern. More often than not, an analyst may want to see not only the outliers detected, but also insightful explanations about the outliers. Particularly, an analyst may want to know, for an outlier,

\begin{abstract}
${ }^{*}$ This is a preliminary and introductory version. Limited by space, several major results in the full paper are omitted. Please refer to the full paper for the complete details. This research is supported in part by an NSERC Discovery Grant, a BCFRST NRAS Endowment Research Team Program project, and a GRAND NCE project. Work by Guozhu Dong was supported in part by NSF grant IIS1044634. All opinions, findings, conclusions and recommendations in this paper are those of the authors and do not necessarily reflect the views of the funding agencies.
\end{abstract}

Permission to make digital or hard copies of all or part of this work for personal or classroom use is granted without fee provided that copies are not made or distributed for profit or commercial advantage and that copies bear this notice and the full citation on the first page. Copyrights for components of this work owned by others than ACM must be honored. Abstracting with credit is permitted. To copy otherwise, or republish, to post on servers or to redistribute to lists, requires prior specific permission and/or a fee. Request permissions from Permissions@acm.org.

SSDBM '13, July 29 - 31 2013, Baltimore, MD, USA

Copyright 2013 ACM 978-1-4503-1921-8/13/07 \$15.00 a reference group of objects from which the outlier deviates in some aspects and shares similarity with in some other aspects, and a set of features manifesting the outlier's unusual/deviating behavior, the outlier degree, and the other similar outliers sharing the same context. Such contextual information can help an analyst to better understand and investigate individual outliers and propose action plans suitable for such outliers. We argue that the contextual information about outliers should be an integral component in the outlier detection process. Unfortunately, most of the existing outlier detection methods do not provide rich and detailed contextual information for outlier analysis.

In this paper, we tackle the problem of contextual outlier detection on categorical data, and make two main contributions. First, we develop a notion of multidimensional contextual outliers to model the context of an outlier. Intuitively, a contextual outlier is a small group of objects that share similarity on some attributes with a significantly larger reference group of objects, but deviate dramatically from the reference group on some other attributes. An example is: "Among the computer science senior undergraduate students at University $X$, a small group of 3 students not enrolled in the data structure course is an outlier against the reference group of 128 students enrolled in the course." In contextual outlier detection, we identify not only the outliers, but also their associated contextual information including (1) comparing to what reference group of objects the detected object(s) is/are an outlier; (2) the attributes defining the unusual behavior of the outlier(s) compared against the reference group; (3) the population of similar outliers sharing the same context; and (4) the outlier degree, which measures the population ratio between the reference normal group and the outlier group. Second, we design a simple yet effective contextual outlier detection algorithm that leverages the state-of-the-art data cube computation techniques. The focus of our method is to find outliers together with their contextual information. We conduct experiments to evaluate the feasibility and usefulness of our approach.

The rest of the paper is organized as follows. We propose the notion of contextual outliers in Section 2, and develop a detection algorithm in Section 3. We evaluate our approach 
in Section 4. We review related work in Section 5, and conclude the paper in Section 6.

\section{CONTEXTUAL OUTLIERS}

In this paper, we consider outlier detection on multidimensional categorical data. Specifically, we consider a base table $T\left(A_{1}, \ldots, A_{n}\right)$, where $A_{1}, \ldots, A_{n}$ are categorical attributes with finite domains. We assume that each object is represented by a tuple in the base table and is associated with an identifier tid, which is used as a reference to the object only, and does not carry any other meaning. For an object $t \in T$, denote by $t . A_{i}$ and t.tid the value of $t$ on attribute $A_{i}(1 \leq i \leq n)$ and the identifier of $t$, respectively.

A subspace is a subset of attributes. In order to summarize a group of objects, we add a wildcard meta-symbol $*$ to the domain of every attribute $A_{i}(1 \leq i \leq n)$. Symbol $*$ matches any possible values in the domain. A group-by tuple (or group for short) is a tuple $g=\left(g \cdot A_{1}, \ldots, g \cdot A_{n}\right)$ such that $g . A_{i}$ takes either a value in the domain of $A_{i}$ or meta-symbol *. The cover of $g$ is the set of objects in $T$ matching $g$, that is, $\operatorname{cov}(g)=\left\{t \in T \mid\right.$ for each $i(1 \leq i \leq n), t . A_{i}=g \cdot A_{i}$ holds whenever $\left.g \cdot A_{i} \neq *\right\}$. The set $\operatorname{space}(g)=\left\{A_{i} \mid 1 \leq\right.$ $\left.i \leq n, g \cdot A_{i} \neq *\right\}$ is called the subspace of $g$, and the set $\operatorname{avs}(g)=\left\{A_{i}=g \cdot A_{i} \mid 1 \leq i \leq n, g \cdot A_{i} \neq *\right\}$ is called the non-* attribute-value set (AVS for short) of group $g$. For an AVS $V$, we overload the operator space $(\cdot)$ by defining $\operatorname{space}(V)=\left\{A_{i} \mid A_{i}\right.$ occurs in $\left.V\right\}$. Thus, space $(\operatorname{avs}(g))=$ $\operatorname{space}(g)$.

For two distinct groups $g_{1}$ and $g_{2}, g_{1}$ is an ancestor of $g_{2}$, and $g_{2}$ a descendant of $g_{1}$, denoted by $g_{1} \succ g_{2}$, if $\operatorname{avs}\left(g_{1}\right) \subset \operatorname{avs}\left(g_{2}\right)$, that is, for every attribute $A_{i}(1 \leq i \leq n)$ such that $g_{1} . A_{i} \neq *$, we have $g_{1} \cdot A_{i}=g_{2} . A_{i}$. We write $g_{1} \succeq g_{2}$ if $g_{1} \succ g_{2}$ or $g_{1}=g_{2}$.

Property 1 (Monotonicity). For two groups $g_{1}$ and $g_{2}$ such that $g_{1} \succ g_{2}, \operatorname{cov}\left(g_{1}\right) \supseteq \operatorname{cov}\left(g_{2}\right)$.

Intuitively, for a group of outlier objects, the contextual information consists of a group of reference objects that manifest the outlier group in a subspace. The comparison of the two groups in population size is also included.

Definition 1 (Contextual OUtlier). Let $T$ be $a$ base table, and $r, o$ be two groups such that space $(r)=$ space $(o) \neq \emptyset$. Given an outlier degree threshold $\Delta>1$, the pair $(r, o)$ is a contextual outlier if the outlier degree $\operatorname{deg}(r, o)=\frac{|\operatorname{cov}(r)|}{|\operatorname{cov}(o)|} \geq \Delta$. We call $r$ the reference group, o the outlier group, out $(r, o)=\operatorname{space}(r)-\operatorname{space}(\operatorname{cond}(r, o))$ the outlier subspace, and cond $(r, o)=\operatorname{avs}(r) \cap \operatorname{avs}(o)$ the shared $\boldsymbol{A} \boldsymbol{V} \boldsymbol{S}$. It is possible that cond $(r, o)$ is empty.

The shared AVS cond $(r, o)$ provides a context subspace for the outlier analysis about $o$. The objects in groups $o$ and $r$ belong to the same context subspace, that is, they take the same values on those attributes that occur in $\operatorname{cond}(r, o)$. If $\operatorname{cond}(r, o)=\emptyset, r$ and $o$ do not share any common features. In such a special case, $o$ is a global outlier that is small in population and different from a large reference group $r$ in space $\operatorname{space}(o)=\operatorname{space}(r)$.
The reference group $r$ indicates the normal or dominating objects to which $o$ is compared. The outlier group $o$ and the outlier subspace out $(r, o)$ indicate the outlier objects $\operatorname{cov}(o)$ and the attributes that manifest the deviation of $o$ from $r$. The outlier degree measures how exceptional the group $o$ is when compared to $r$. The larger the outlier degree is, the more outlying $o$ is.

\section{DETECTION ALGORITHMS}

In this section, we develop an algorithm for contextual outlier detection. We observe that group-bys are essential units in both data cube computation and contextual outlier analysis, so we can exploit the state-of-the-art data cube techniques in detecting contextual outliers.

Lemma 1 (Non-Closure ATtRibutes). For two contextual outliers $\left(r_{1}, o_{1}\right)$ and $\left(r_{2}, o_{2}\right)$ in a base table $T$, if $r_{1} \succ r_{2}, o_{1} \succ o_{2}, \operatorname{cov}\left(r_{1}\right)=\operatorname{cov}\left(r_{2}\right)$, and $\operatorname{cov}\left(o_{1}\right)=\operatorname{cov}\left(o_{2}\right)$, then $\operatorname{deg}\left(r_{1}, o_{1}\right)=\operatorname{deg}\left(r_{2}, o_{2}\right)$.

In the two contextual outliers $\left(r_{1}, o_{1}\right)$ and $\left(r_{2}, o_{2}\right)$ in Lemma 1, the two groups $r_{1}$ and $r_{2}$ capture the same set of objects. Hence $\left(r_{1}, o_{1}\right)$ is redundant given $\left(r_{2}, o_{2}\right)$ or vice versa. Since $r_{1} \succ r_{2}, r_{2}$ contains some extra attributes in addition to those in $r_{1}$. Hence $r_{2}$ is more informative and descriptive than $r_{1}$ as a reference group. It is better to include $\left(r_{2}, o_{2}\right)$ for outlier analysis.

Definition 2 (Closure Group/OUtlier). Given a base table $T$, a group $g$ is a closure group if for any descendant group $g^{\prime} \prec g, \operatorname{cov}\left(g^{\prime}\right) \subset \operatorname{cov}(g)$. $(r, o)$ is called a closure outlier if there does not exist another contextual outlier $\left(r^{\prime}, o^{\prime}\right)$ such that $r^{\prime} \prec r, o^{\prime} \prec o, \operatorname{cov}(r)=\operatorname{cov}\left(r^{\prime}\right)$, and $\operatorname{cov}(o)=\operatorname{cov}\left(o^{\prime}\right)$.

Theorem 1 (Closure Group/outlier). Contextual outlier $(r, o)$ is a closure outlier if and only if either $r$ or $o$ is a closure group.

Since every closure contextual outlier must have either the reference group or the outlier group as a closure group, we can find all closure groups in the base table first, and then use the closure groups to find contextual outliers. Finding closure groups and closure patterns has been well studied in frequent pattern mining $[11,15]$ and data cube computation [7]. Given a base table $T$, we can adopt a state-of-theart algorithm, such as the DFS algorithm in [7], to find all closure groups.

Algorithm 1 presents the pseudocode of our detection method, COD (for Contextual Outlier Detection). For each closure group $o$, we consider all the other closure groups $r$ such that $(r, o)$ is a contextual outlier. Obviously, $|\operatorname{cov}(o)|$ cannot be larger than $\frac{l}{\Delta}$, where $l$ is the largest cover size among all closure groups (calculated in Line 1). For each of such closure groups $o$, we iterate over all the other closure groups $r$ such that $|\operatorname{cov}(r)| \geq \Delta|\operatorname{cov}(o)|$ (the inner loop, Lines 5-7). The iteration continues until all closure groups that may be outlier groups are examined. 


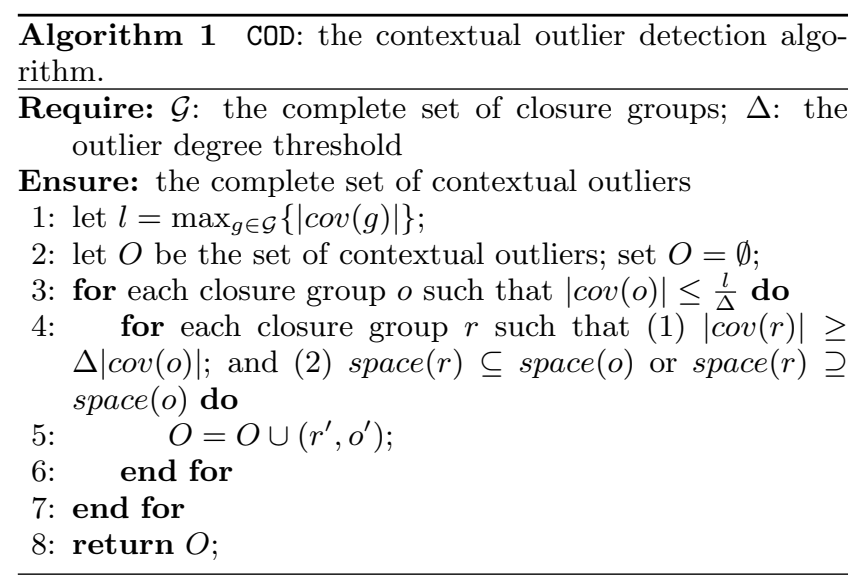

\begin{tabular}{|c|c|c|c|c|}
\hline $\begin{array}{c}\text { Data } \\
\text { Set }\end{array}$ & $\begin{array}{c}\text { Solar- } \\
\text { flare }\end{array}$ & $\begin{array}{c}\text { Tic- } \\
\text { tac-toe }\end{array}$ & $\begin{array}{c}\text { Credit- } \\
\text { approval }\end{array}$ & $\begin{array}{c}\text { Hayes- } \\
\text { roth }\end{array}$ \\
\hline \hline \# obj. & 1,389 & 958 & 690 & 160 \\
\hline \# attr. & 10 & 9 & 8 & 4 \\
\hline \# closure grp. & 7,770 & 42,711 & 5,707 & 277 \\
\hline QC time (s) & 2.136 & 12.903 & 1.446 & 0.047 \\
\hline
\end{tabular}

Note: QC time refers to the time used to find all closure groups, that is, $|\mathcal{G}|$ in algorithm 1.

Table 1: The statistics of the data sets.

\section{EXPERIMENTAL RESULTS}

In this section, we report our empirical evaluation of COD using real data sets. All experiments were conducted on a PC computer with an Intel Core Duo E8400 3.0 GHz CPU and 4 GB main memory, running the Microsoft Windows 7 operating system. The algorithms were implemented in C++ using Microsoft Visual Studio 2010.

We cannot identify any existing method that solves the exact same problem. The focus of our method is to find outliers with contextual information. Consequently, this paper does not intend to compete with the existing methods.

We use categorical data sets from the UCI repository [5]. We report the results on four data sets: solar-flare, tic-tactoe, credit-approval, and hayes-roth. Some statistics of the data sets are summarized in Table 1 . We evaluate COD in two aspects: effectiveness and efficiency.

\subsection{Case Studies}

We demonstrate the effectiveness of context outlier detection using case studies on the data set hayes-roth.

The hayes-roth data set records the information about 160 people on four attributes $[1,5]$. The first attribute, hobby, takes values uniformly at random [5] and we thus ignore it in our analysis, that is, all groups take value $*$ on the attribute. The description for the other three attributes are adopted from [1]: the second attribute, age, takes values in $\{30,40,50, r>0\}$ (the meaning of value " $r>0$ " is unspecified in [1]); attribute education takes values in fjunior-high, high-school, trade-school, college $\}$; and the last attribute, marital-status, takes values in single, married, divorced,

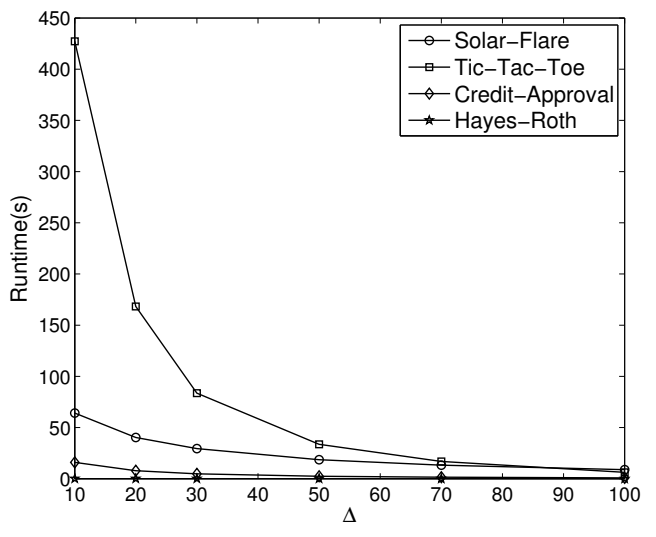

Figure 1: The runtime of COD on the four real data sets.

widowed $\}$. Table 2 shows some interesting contextual outliers with respect to $\Delta=5$.

In Table 2 , outliers $c_{1}$ and $c_{2}$ share the same reference group. The reference group consists of 34 people whose maritalstatus is "single" and who have high-school degrees. Outlier group $c_{1}$ is a collection of 6 "divorced" college graduates and outlier group $c_{2}$ is a collection of 6 "single" trade school graduates. Outlier $c_{5}$ is interesting: among people who are divorced, those who are college graduates are outliers compared to those with high school degrees. $c_{9}$ shows that, among people who are 50-years old, the 2 with college degrees are outliers compared to the 16 with high school degrees. Another interesting outlier is $c_{10}$ : in the age group of 30 , the 4 people widowed are outliers compared to the 34 people married. Please note that, in the whole data set, there are 59 of high-school, 59 of junior-school, 29 of tradeschool and 13 of college graduates. Given $\Delta=5$, those of trade-school and college graduates are not outliers comparing to those of high-school and junior-school. The outliers can only be explained well using the contextual information.

\subsection{Efficiency}

COD takes only one parameter, the outlier degree threshold $\Delta$. Figure 1 shows the runtime of COD on the four real data sets with respect to various $\Delta$ thresholds. The closure group computation time is reported in Table 1 , and is not included.

\section{RELATED WORK}

To the best of our knowledge, only very few existing studies consider context in outlier detection. Song et al. [13] proposed the notion of conditional outliers to model the outliers manifested by a set of behavioral attributes (e.g. temperature) conditionally depending on a set of contextual attributes (e.g. longitude and latitude). The behavioral attributes and the contextual attributes are pre-defined. Our contextual outlier model automatically identifies shared AVSs. Moreover, reference groups are not modeled in [13]. A mixture model is used in [13] to mine conditional outliers, which is infeasible in our model since here the subspaces are not pre-defined and change from one outlier to another. Valko et al. [14] detected conditional anomalies using a training set of labeled examples with possible label noise, which is different from our work that no labeled data is assumed. 


\begin{tabular}{|c|c|c|c|}
\hline Outlier-id & Reference group $r$ & Outlier group $o$ & $\operatorname{deg}(r, o)=\frac{|\operatorname{cov}(r)|}{|\operatorname{cov}(o)|}$ \\
\hline \hline$c_{1}$ & $(*, *$, high-school, single $)$ & $(*, *$, high-school, divorced $)$ & $5.7=34 / 6$ \\
$c_{2}$ & $(*, *$, high-school, $\underline{\text { single })}$ & $(*, *$, trade-school, single $)$ & $5.7=34 / 6$ \\
$c_{3}$ & $(*, *$, high-school, single $)$ & $(*, *$, high-school, widowed $)$ & $8.5=34 / 4$ \\
$c_{4}$ & $(*, *$, trade-school, married $)$ & $(*, *$, trade-school, widowed $)$ & $8.0=16 / 2$ \\
$c_{5}$ & $(*, *$, junior-high, divorced $)$ & $(*, *$, college, divorced $)$ & $8.0=16 / 2$ \\
$c_{6}$ & $(*, \underline{40}$, junior-high, $*)$ & $(*, \underline{40}$, college, $*)$ & $8.5=34 / 4$ \\
$c_{7}$ & $(*, \underline{40}$, junior-high, $*)$ & $(*, \underline{40}$, trade-school, $*)$ & $5.7=34 / 6$ \\
$c_{8}$ & $(*, \underline{40}$, junior-high, $*)$ & $(*, 50$, junior-high, $*)$ & $5.7=34 / 6$ \\
$c_{9}$ & $(*, \underline{50}, \underline{\text { high-school, } *)}$ & $(*, \underline{50, \text { college, } *)}$ & $8.0=16 / 2$ \\
$c_{10}$ & $(*, \underline{30}, *$, married $)$ & $(*, \underline{30,}, *$, widowed $)$ & $8.5=34 / 4$ \\
\hline
\end{tabular}

Table 2: Some contextual outliers on data set hayes-roth. The underlined attributes indicate the shared AVSs.

Kriegel et al. [6] proposed a method that detects an outlier with reference to the axis parallel subspace spanned by its neighbors. Müler et al. [10] proposed a technique for ranking outliers based on their degree of deviation in different subspace projections. While these studies also focus on subspace context for outliers, there are two key differences from our work. First, these studies focus on continuous datasets, while our focus is on categorical relational data. Second, our work proposes techniques for concise descriptions of sets of outliers. We also provide contextual descriptions for the outliers that are detected.

Recently, Smet and Vreeken [12] developed an outlier detection method $O C^{3}$, which assumes that outliers are generated by a distribution different from that generates the normal objects, and uses minimum description length (MDL) to detect outliers. Again, the notions of context, reference groups and outlier groups are not modeled simultaneously in $O C^{3}$. Angiulli et al. [2] studied a related by orthogonal problem. Given a multidimensional database and a query object in the database, find the top- $k$ subset of attributes that the query object receives the highest outlier score. Their method does not find outliers directly. Moreover, it finds subspaces but does not find reference groups in outlier explanation.

A contextual outlier $(r, o)$ identified in our method can be written as a pair of rules: $\operatorname{cond}(r, o) \Rightarrow \operatorname{avs}(r)-\operatorname{cond}(r, o)$ for the reference group, and $\operatorname{cond}(r, o) \Rightarrow \operatorname{avs}(o)-\operatorname{cond}(r, o)$ for the outlier group. There are a number of methods using rules in outlier detection $[4,9,16]$. Our method differs from the existing methods in several aspects. First, most of the existing rule-based methods focus on detecting individual outliers, and may not be able to identify outlier groups and measure the outlyingness accordingly. Second, many existing rule-based methods use rules to model only the normal objects or strong associations. Outliers are individual objects that do not follow those rules. Those methods do not model and analyze context explicitly. Lastly, many existing methods, such as $[3,8,16]$, set strict constraints on the size of the rules or the aggregate groups to be considered, such as a very small number of items/attributes allowed in a rule or only the parents and their sibling groups.

\section{CONCLUSIONS AND FUTURE WORK}

This paper represents the first step in an ambitious journey towards contextual outlier detection and analysis. We proposed a framework for contextual outlier detection. Our focus was to improve the interpretability of outliers. In particular, we argued that the context of an outlier should include a shared AVS, a reference group, an outlier group, and an outlier degree measure. We developed a detection algorithm leveraging the state-of-the-art data cube computation techniques.

\section{REFERENCES}

[1] J.-R. Anderson and P.-I. Kline. A learning system and its psychological implications. In IJCAI, 1979.

[2] F. Angiulli, et al. Detecting outlying properties of exceptional objects. ACM Trans. Database Syst., 2009.

[3] K. Das and J. Schneider. Detecting anomalous records in categorical datasets. In $K D D, 2007$.

[4] T. Fawcett and F. Provost. Adaptive fraud detection. Data Min. Knowl. Discov., 1997.

[5] A. Frank and A. Asuncion. UCI machine learning repository, 2010.

[6] H.-P. Kriegel, et al. Outlier detection in axis-parallel subspaces of high dimensional data. In PAKDD, 2009.

[7] L.-V.-S. Lakshmanan, et al. Quotient cube: how to summarize the semantics of a data cube. In $V L D B$, 2002.

[8] S. Lin and D.-E. Brown. An outlier-based data association method for linking criminal incidents. Decis. Support Syst., 2006.

[9] M.-V. Mahoney and P.-K. Chan. Learning rules for anomaly detection of hostile network traffic. In ICDM, 2003.

[10] E. Müller, et al. Statistical selection of relevant subspace projections for outlier ranking. In $I C D E$, 2011.

[11] N. Pasquier, et al. Discovering frequent closed itemsets for association rules. In ICDT, 1999.

[12] K. Smets and J. Vreeken. The odd one out: Identifying and characterising anomalies. In SDM, 2011.

[13] X. Song, et al. Conditional anomaly detection. IEEE Trans. on Knowl. and Data Eng., 2007.

[14] M. Valko, et al. Conditional anomaly detection with soft harmonic functions. In ICDM, 2011.

[15] J. Wang, et al. Closet+: searching for the best strategies for mining frequent closed itemsets. In $K D D, 2003$.

[16] W.-K. Wong, et al. Rule-based anomaly pattern detection for detecting disease outbreaks. In ENAI, 2002 . 